\title{
OTIMIZAÇÃO DE PROTOLOCOS DE AMOSTRAGEM PARA MINÉRIO DE ALUMÍNIO
}

D.A. BORTOLETO", A. C. CHIEREGATI, R. C OLIVEIRA

*Universidade de São Paulo, Escola Politécnica - São Paulo SP

bortoda@usp.com

Submetido 09/10/2017 - Aceito 08/11/2017

DOI: $10.15628 /$ holos.2017.6379

\section{RESUMO}

A definição do protocolo de amostragem apropriado é de extrema importância para qualquer operação mineira, em especial a do minério de alumínio, devido ao controle rigoroso da qualidade do produto final. A estratégia para obtenção dos dados necessários para garantia e controle da qualidade (QAQC) deve levar em consideração contribuições multidisciplinares que incluem estatística, geoestatística, geologia e a teoria da amostragem (theory of sampling, ou TOS), fundamentais para se estabelecerem metas de precisão e acurácia considerando-se os principais constituintes do minério de alumínio: alumina disponível e sílica reativa. Selecionar um protocolo de amostragem adequado requer a estimativa experimental das heterogeneidades de constituição e de distribuição para gerenciamento contínuo, além da perseverante aplicação dos conceitos da TOS para eliminação de todas as possíveis fontes de viés. O presente trabalho apresenta uma proposta alternativa e complementar para a normatização de procedimentos corretos de amostragem de minério de alumínio, com base na otimização de protocolos de amostragem segundo a teoria da amostragem para minérios granulados de Pierre Gy.

\section{OPTIMIZATION OF SAMPLING PROTOCOLS FOR ALUMINIUM ORE}

\section{ABSTRACT}

The controlled stability of concentrated aluminium ore is a crucial problem when there is no defined sampling protocol. The strategy to obtain the necessary data for an effective Quality Assurance and Quality Control (QA-QC) program have to be taken into account. The input from several disciplines including Statistics, Geostatistics, Geology and Sampling are needed to establish realistic goals for accuracy and precision levels regarding the main aluminium ore constituents: available alumina and reactive silica. Selecting an appropriate sampling protocol requires the estimation of the constitutional and distributional heterogeneities and the application of the Theory of Sampling in order to eliminate all bias generators. The international sampling standards describe tests to evaluate precision and accuracy to improve data reliability, however, they are unclear and incomplete. The present paper presents a proposal of optimising sampling protocols for aluminium ore based on current standards procedures, Pierre Gy's Theory of Sampling and geostatistical concepts.

KEYWORDS: aluminium ore; sampling protocol; heterogeneity 


\section{INTRODUÇÃO}

Um programa de garantia e controle da qualidade, QAQC (quality assurance and quality control) em um empreendimento mineiro deve ser implantado de forma robusta, com base não apenas em princípios da estatística clássica, mas também na geoestatística e principalmente na teoria da amostragem de materiais fragmentados de Pierre Gy (TOS). Existe uma ligação muito forte entre a geoestatística e a TOS, de modo que uma não pode ser compreendida em sua totalidade sem a outra (FRANÇOIS-BONGARCON, 2004).

Existem hoje diversos esforços para promover a TOS nos ciclos acadêmicos e nas áreas técnicas da indústria. Entretanto, a amostragem ainda é um assunto negligenciado pelas operações mineiras devido à falta de conhecimento da existência de uma teoria, principalmente dos elevados níveis hierárquicos das empresas, que, frequentemente, não fazem os investimentos necessários em equipamentos e treinamentos.

As normas internacionais de amostragem existentes são deficientes e se baseiam apenas na estatística clássica (ou seja, a estatística de variáveis aleatórias) para o tratamento de dados oriundos de fenômenos naturais, que não são estritamente aleatórios. Para materiais naturais, que é o caso dos minérios, deve-se lançar mão de técnicas de geoestatística e da TOS a fim de se estimar corretamente a variância destes processos. Uma vez lavrado, o minério deve ser amostrado periodicamente em etapas estratégicas do processo, respeitando-se uma série de critérios, que incluem: massa mínima representativa de amostras; frequência da obtenção dos incrementos; características do sistema de amostragem para garantir amostras não-enviesadas; preparação dos incrementos; gerenciamento de técnicas analíticas.

\section{METODOLOGIA}

O primeiro passo para otimização do protocolo de amostragem é a determinação da massa mínima de amostra. Recomenda-se o uso do teste de heterogeneidade proposto por Pitard (1993). Utilizou-se as seguintes faixas granulométricas para o estudo: "-38,1 mm; +25,4 mm", "-25,4 mm; +12,7 mm"; “-12,7mm; +6,3mm"; “-6,3mm; +1,2mm". Desta forma, se estimou o fator constante de heterogeneidade EST IHL através da equação 1.

$$
\operatorname{EST~IH} H_{L}=g \sum \frac{\left(a_{q}-a_{Q}\right)^{2}}{a_{Q}^{2}} \cdot \frac{M_{q}^{2}}{M_{Q}}
$$

A correlação linear entre a constante de heterogeneidade e os diâmetros (d95) de cada uma das quatro faixas granulométricas avaliadas, permite a calibração da fórmula de Gy para estimativa do erro fundamental de amostragem (FSE), assim como as massas mínimas de amostra. 
O segundo passo consiste na determinação do intervalo entre os incrementos. Neste caso, a Geoestatística é a única ciência que permite esta estimativa através da variografia, baseada em modelo contínuo em relação ao tempo (GY, 1998). Uma vez os incrementos obtidos em ordem cronológica, o variograma é obtido através da equação 2.

$$
v(j)=\frac{1}{2 N} \sum_{q}\left(t_{q+j}-t_{q}\right)^{2}
$$

A área abaixo do variograma, estimada por integral dupla, representa o erro de flutuação da heterogeneidade (HFE) que passa a ser referência para aceitação de intervalos maiores ou menores entre os incrementos.

Testes de viés podem ser executados em sistemas de amostragem para finalidade de certificação. Entretanto, uma serie de parâmetros referentes ao design deste sistema deve ser levado em consideração a fim de se eliminar os erros de amostragem geradores do viés: erro de delimitação do incremento (IDE); erro de extração do incremento (IEE); erro de preparação do incremento (IPE). Na Figura 1 está representado um exemplo de sistema que não apresenta IDE, onde a espessura do corte do fluxo cadente de fragmentos é constante.

\section{Espessura do corte} constante

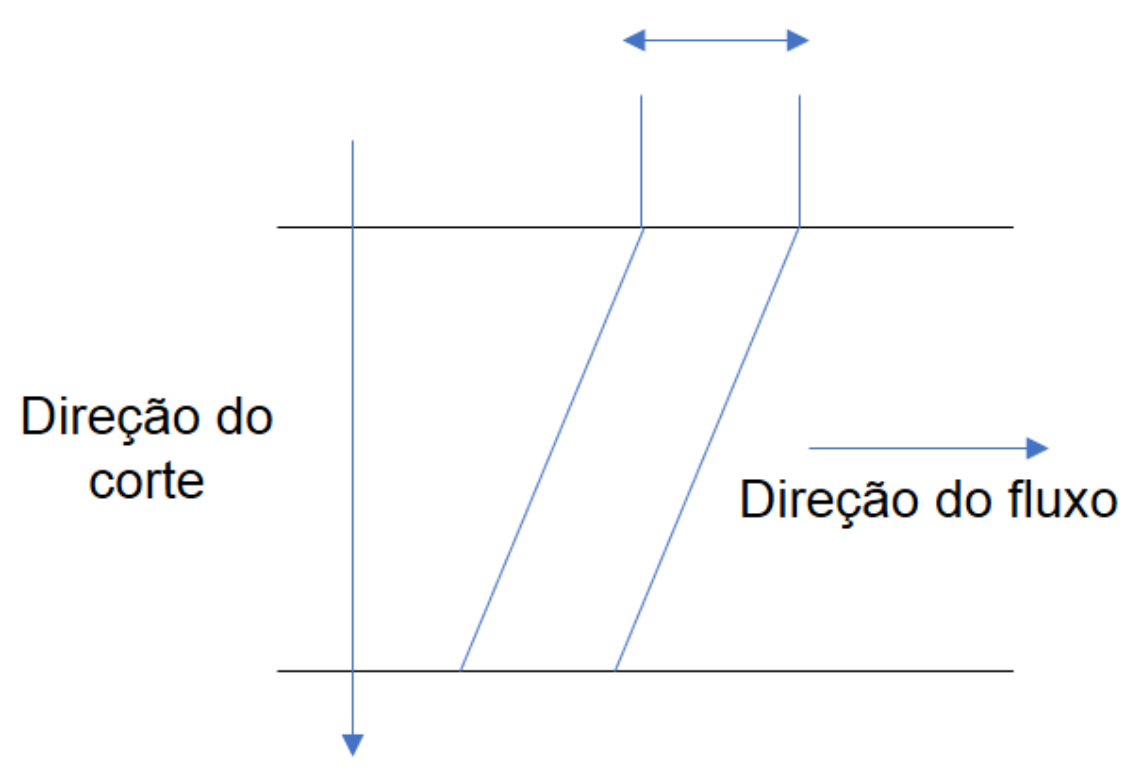

Figura 1. Erro de delimitação do incremento (IDE). (Fonte: Pitard 2015) 
Na Figura 2 está representado o design ideal do cortador a fim de se garantir a eliminação do IEE.

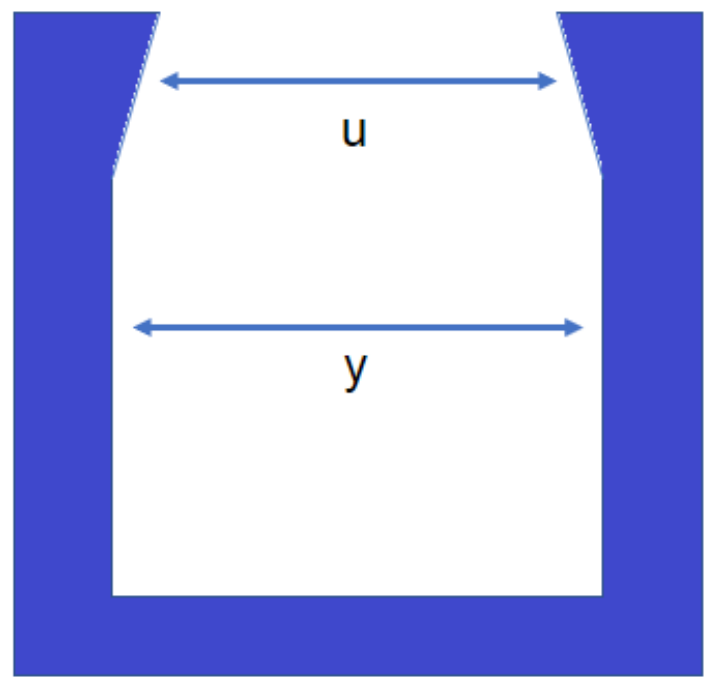

Figura 2. Erro de extração do incremento (IEE). (Fonte: Pitard 2015)

Neste caso, a abertura $u$ deve ser ao menos 3 vezes maior que o "top size" dos fragmentos amostrados. Em relação ao IPE, deve-se ter uma série de cuidados nas etapas de preparação como o uso de métodos de divisão adequado e evitar perdas ou trocas de amostras.

\section{RESULTADOS E DISCUSSÃO}

A correlação linear entre a constante de heterogeneidade e o diâmetro (d95) das faixas granulométricas avaliadas está representada na Figura 3.

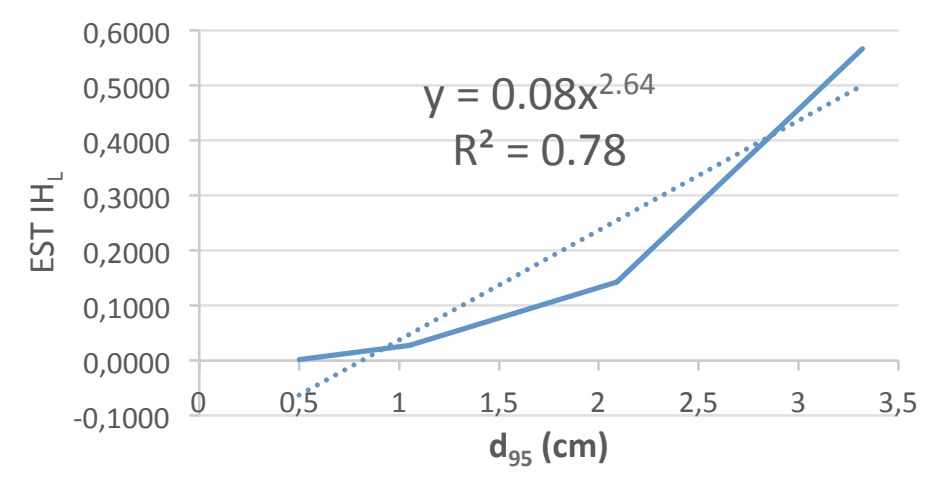

Figura 3. Estimativa da constante de heterogeneidade 
Conforme mencionado anteriormente, a fórmula de Gy foi calibrada com base está correlação linear de acordo com Equação 3.

$$
S_{F S E}^{2}=0,08 \cdot d_{95}^{2.64} \cdot\left(\frac{1}{M_{S}}-\frac{1}{M_{L}}\right)
$$

Onde $d_{95}$ é o diâmetro pelo qual $95 \%$ do material é passante, Ms a massa da amostra, $\mathrm{ML}$ a massa do lote e $s_{F S E}^{2}$ é o desvio padrão relativo referente ao FSE. Os resultados de massa mínima de amostra estão na Figura 4, onde assumiu-se FSE máximo igual a 3\% (desvio padrão relativo).

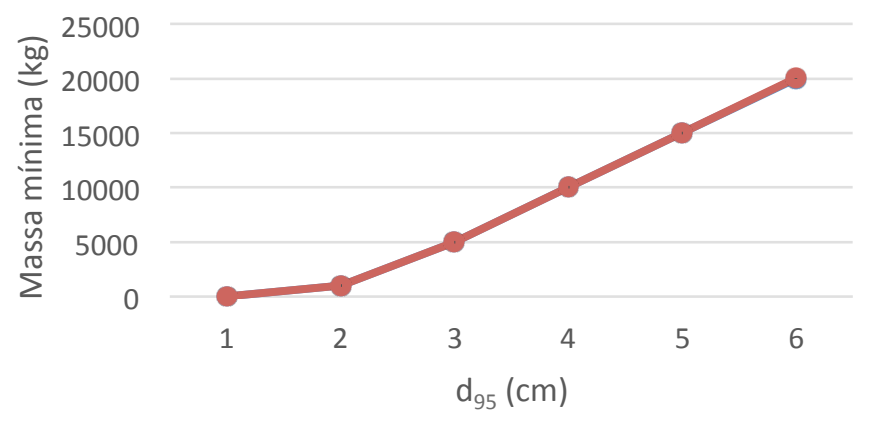

Figura 4. Massa mínima de amostra

O variograma obtido a partir de incrementos de forma cronológica está representado na Figura 5, onde j representa o intervalo mínimo adotado de 1000 toneladas obtendo-se 0,496\% (desvio padrão relativo) para o erro de flutuação do incremento (HFE). 


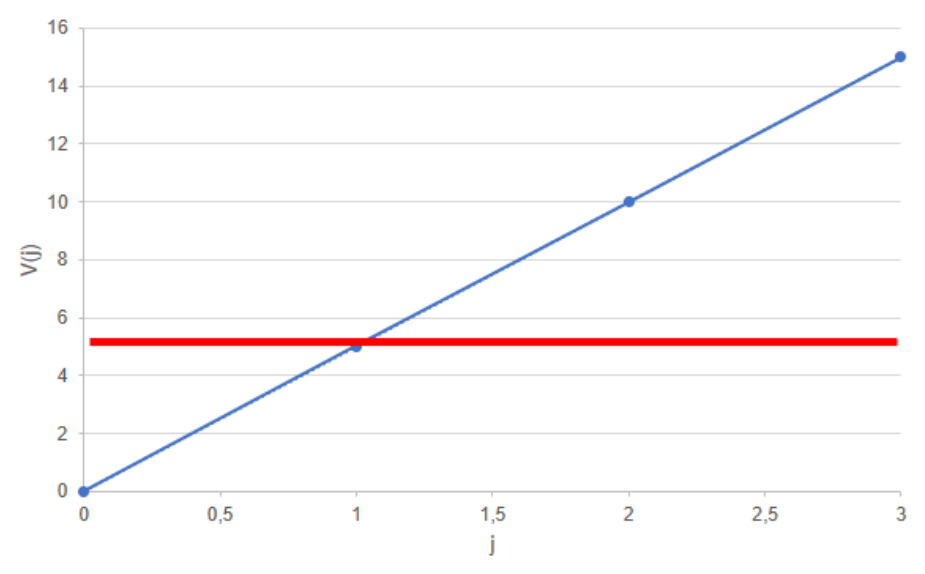

Figura 5. Variograma

\section{CONCLUSÕES}

A teoria da amostragem (TOS) definitivamente deve ser levada em consideração nas normas de amostragem do setor mineral. Especificamente no presente trabalho, sua importância foi demonstrada através de fundamentação teórica e procedimentos experimentais utilizando-se o minério de alumínio como matéria prima. Recomenda-se que estimativa dos erros de amostragem seja realizada em todas as etapas do empreendimento mineiro através da metodologia proposta, que associa conceitos estatísticos à TOS.

O menor entre todos os erros de amostragem, o erro fundamental de amostragem, deve ser estimado visando ao controle estatístico de processos (CEP), à definição de protocolos ótimos nos laboratórios e à determinação de massas mínimas representativas em todas as etapas de amostragem.

A contribuição do erro de segregação e agrupamento (GSE) estimada neste trabalho é elevada, mas pode ser muito maior, caso a metodologia empregada, especialmente nas etapas de divisão de amostras, não seja adequada quanto ao número de incrementos das subamostras obtidas.

O erro de flutuação da heterogeneidade (HFE) deve ser conhecido e levado em consideração para se determinar a periodicidade ótima da retirada de incrementos. Muitas vezes, devido ao grande número de amostras geradas, existe a pressão por parte da operação para reduzir a frequência de amostragem. Neste contexto a análise variográfica é uma poderosa ferramenta para se justificar investimentos e o tempo demandado para obtenção de maior número de incrementos para uma estimativa da qualidade do lote de material fragmentado.

Conhece-se, também, a importância do projeto do sistema de amostragem dos cuidados com o manuseio e preparação dos incrementos, especialmente na rotina da mineração. Portanto, recomendam-se práticas corretas e uso de equipamentos corretos de amostragem para se evitarem os erros sistemáticos, quantificados somente por meio de testes trabalhosos, demorados e, muitas vezes, dúbios. 
Assim como para os erros de amostragem, o gerenciamento diário dos métodos analíticos no laboratório é de fundamental importância, visto que o erro analítico (AE) está incluído em todos os resultados das amostras enviadas ao laboratório. Práticas laboratoriais devem sempre priorizar a qualidade dos resultados e não apenas o tempo de entrega dos mesmos. Desta forma, os cuidados e procedimentos experimentais descritos na metodologia proposta, tais como a otimização da temperatura e do tempo de secagem, devem ser levados em consideração.

A otimização de protocolos de amostragem, especialmente no setor mineral não é tarefa simples. O monitoramento dos principais indicadores do processo através de cartas de controle deve ser constante para que ocorra uma efetiva e contínua melhoria do processo. A especificação da qualidade do minério junto ao cliente deve contemplar a soma da variância dos erros gerados em todas as etapas do protocolo de amostragem. Além disso, o conhecimento detalhado das contribuições de cada fonte geradora de erro permite a atuação efetiva nas etapas críticas, evitando que os mesmos erros sejam repetidos no futuro.

Este trabalho visou enriquecer as normas atualmente existentes no que diz respeito a procedimentos de amostragem e à verificação de acurácia e precisão de sistemas de amostragem. Os experimentos apresentados foram realizados com amostras de minério de alumínio da região de Juruti, no oeste do Pará. Os princípios teóricos aqui detalhados e os testes aqui propostos são aplicáveis tanto para bauxita de outras regiões quanto para outros tipos de minério. Entretanto, as conclusões relacionadas aos resultados dos testes realizados com o minério de Juruti não devem ser tomadas como verdadeiras para qualquer tipo de minério. Recomenda-se que os testes propostos no presente trabalho sejam realizados para cada operação mineira e sempre que houver mudanças no projeto do amostrador, no procedimento de preparação de amostras, ou nas características do minério.

\section{REFERÊNCIAS}

FRANÇOIS-BONGARÇON, D. (2004). Theory of sampling and geostatistics: an intimate link. Chemometrics and Intelligent Laboratory Systems, v. 74, n. 1, p. 143-148.

GY P.M. (1998). Sampling for analytical purposes. Chichester: Wiley.

PITARD, F.F. Pierre (1993). Gy's sampling theory and sampling practice: heterogeneity, sampling correctness, and statistical process control. $2^{\text {nd }}$. ed. Boca Raton: CRC Press.

PITARD, F.F. (2015). Theory of sampling as applied to process control. Sampling Theory and Methods Course Press, WCSB 7, Bordeaux. 